\title{
High-altitude anti-falling device
}

\author{
Xin Zhang \\ North China Electric Power University, Baoding 071000, China
}

Keywords: High-altitude anti-falling device, OPENCV, pneumatic structure

\begin{abstract}
High-altitude anti-falling device"is a set of automatic device preventing high altitude objects from falling. This automatic device mainly consists of three parts, the falling object detection and recognition system(ODRS), the action control system and the pneumatic structure system. It is a good combination of both electronic technology and mechanical technology. The ODRS detects and recognizes objects. The action control system decides what to do. The pneumatic structure system is execution system to receive the falling objects.
\end{abstract}

\section{Introduction}

High altitude falling matter is a kind of "urban disease”.Now, whether in a high-rise residential district, buildings under construction,or in a high-rise populated place, there are an army of accidents caused by high altitude falling objects.In residential district, maybe a household is not responsible to the throw things freely... The construction site is a high incidence of falling objects zone,for example,no fixed parts or high operating workers is likely to become a precipitating factor.

With people's attention to the problem, relevant departments have taken measures. Common there: installing electronic eyes monitoring equipment to monitor the floor tenants throw outward; developing laws and regulations related to the public; carrying out education and so on.[1]

Due to the frequent occurrence of such events, we had a idea to make a kind of high-altitude anti-falling automatic device. The device ,to a great extent, can protect people's life and property safety, avoiding many unnecessary troubles such as disputes and legal issues after the event .

So the principle, structure and function of the device are introduced in this paper.

\section{Principle and Scheme design}

\subsection{The principle.}

An unidentified object is falling. Then the computer vision library OPENCV recognize it and transform information to processor (IPC). Next IPC processes information and send it to the single chip microcomputer through the serial port. Finally microcomputer control the motor drive the receiving device.

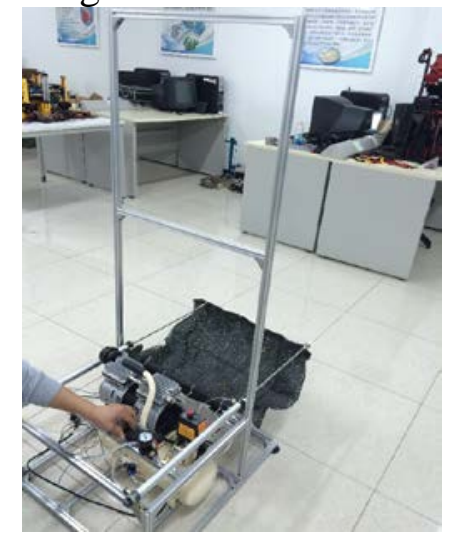

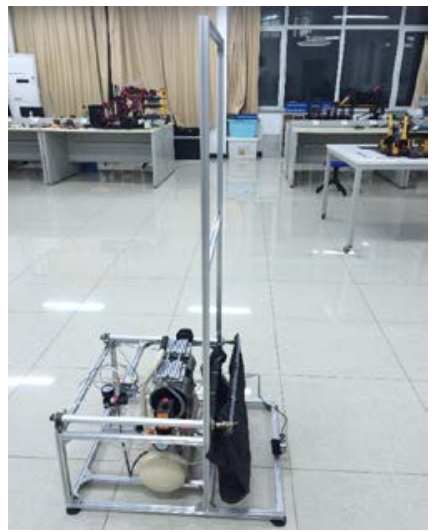

(a)

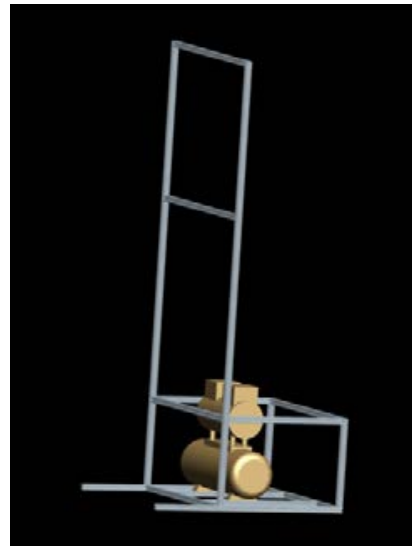

(b)

Fig. 1 High-altitude anti-falling device

a Device physical , b Appearance design 


\subsection{The scheme design.}

1. The first step is to write code of the falling object detection and recognition system.

2. Design and construction of hardware control circuit.

3. Design and construction of mechanical structure.

\section{The falling object detection and recognition system}

\subsection{Introduction.}

The core of this device is the algorithm of OPENCV. Requirements are that to collect data through the USB camera ,using $\mathrm{c}^{++}$combined with the OPENCV library with the computer to process image, detect and identify the high-altitude falling objects, and send data to the microcomputer through the serial port. Meanwhile we developed a high-altitude falling object detection software (HFOD) with a better human-computer interaction.Fig. 2 is the interface and icon of the software.
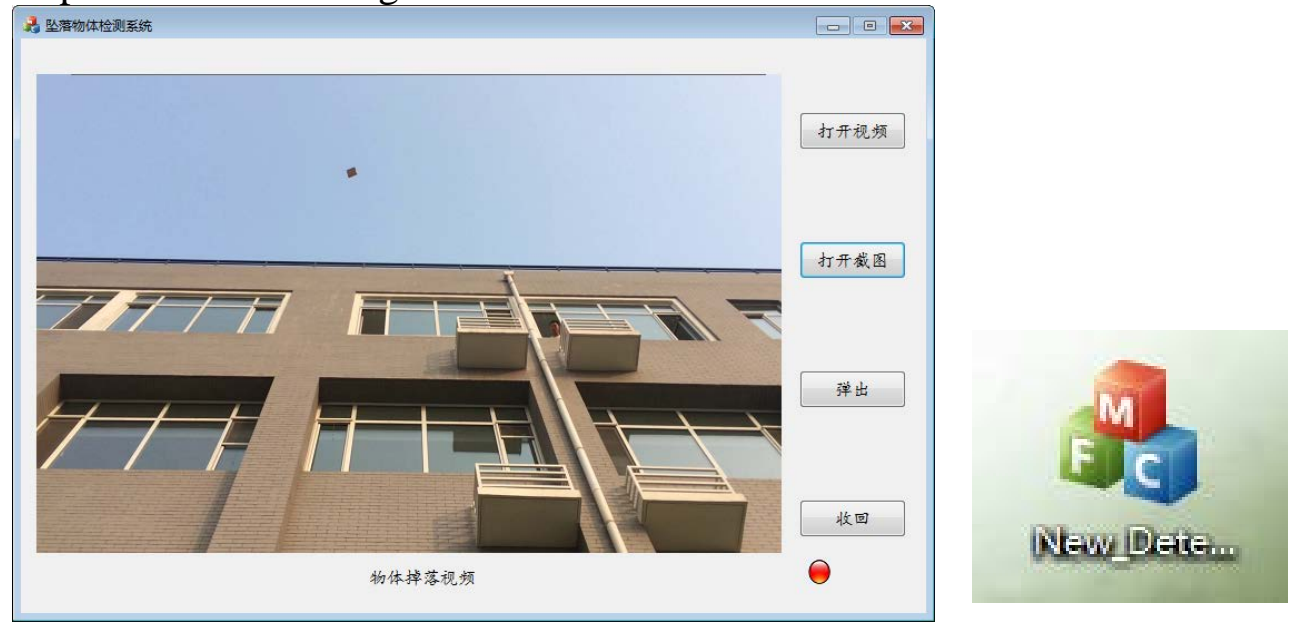

Fig. 2 The interface and icon of HFOD

OPENCV is "Open Source Computer Vision Library". It is a cross platform computer vision library based on (open) issued, operating in Linux, windows or Mac OS operating system. It is lightweight and efficient -- consists of a series of $\mathrm{C}$ function and a small amount of $\mathrm{C}++$ class structure, at the same time provides many interfaces to python, ruby, MATLAB language. It realize the many common algorithms of image processing and computer vision of open source computer vision Library. The high-definition images processed by OPENCV meet the need of our project.[2..3]

\subsection{Algorithm.}

The follows are the current algorithm of high-altitude falling object detection software:

1. Take image acquisition points on the four part of the gray value;

2. Carry on binaryzation;

3. Compare the two matrices with two value. The one matrix is of the last frame,the other is of the fist frame.

4. If a region with continuous pixels changing, detect that there are the falling objects.

\section{The action control system}

After detecting falling objects, the computer send the information to the microcomputer through the serial port . Microcomputer drives pneumatic structure to receive the objects. MCU control core uses a single-chip microcomputer 51, receiving data sent by the computer serial port, and through the $12 \mathrm{~V}$ driving circuit driving electromagnetic valve device.

Figure three is the hardware circuit package and control circuit diagram. MCU we used includes power module, keyboard module, buzzer, LED module, motor driver module, OLED LCD module, 
USB to TTL serial port module, crystal oscillator module, reset module, serial extraction and the smallest single-chip system.
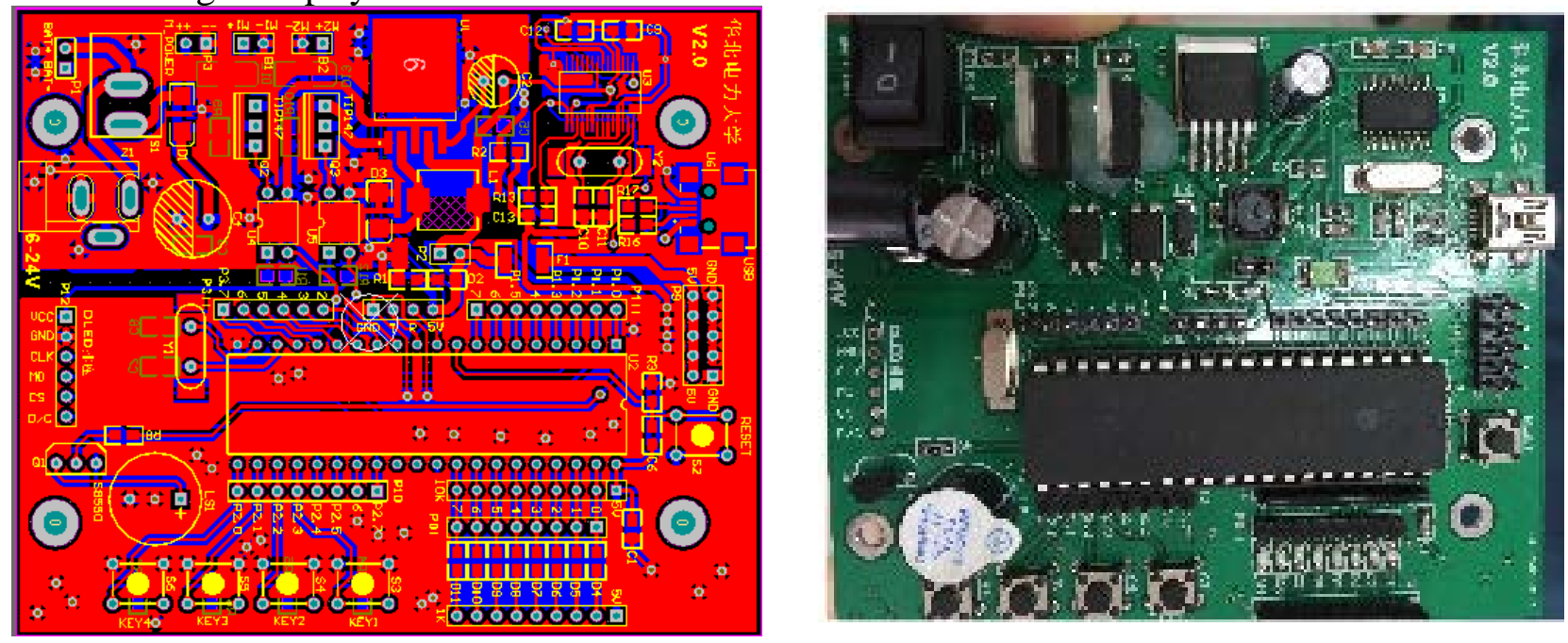

Fig. 3 The hardware circuit package and control circuit diagram

\section{The pneumatic structure system}

\subsection{Principle.}

In order to get a high speed of mechanical and electrical, we use the pneumatic structure. [4] Figure four is the pneumatic circuit diagram . The work of the structure process is described as follows:

This pneumatic structure is popped back through the microcomputer driven cylinder . The motor drums air into pump, pump outputs the gas pressure through the overflow valve. If the output pressure is $0.4 \mathrm{MPa}$, electromagnetic valve received voltage driven signal from MCU. Then the cylinder to drive or recovery the pop-up receiving device.

This pneumatic structure consists of cylinder, motor, pump, reversing solenoid valve, overflow valve and pop-up receiving device.

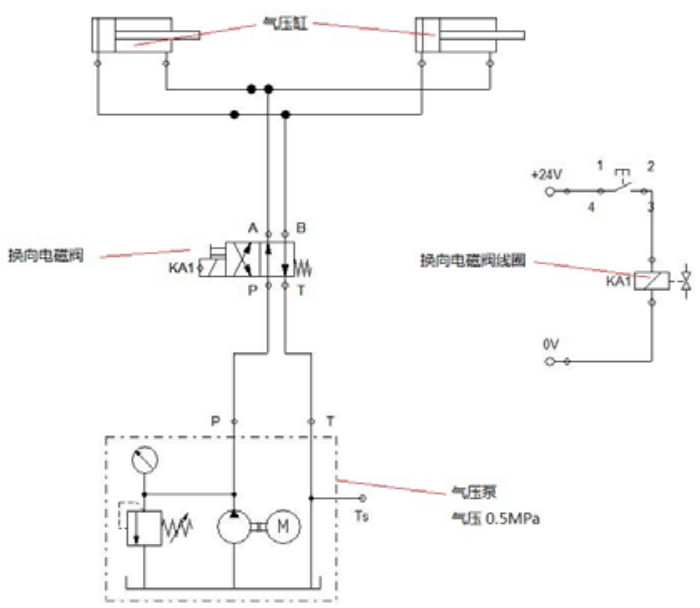

\subsection{Advantages of pneumatic structure.}

Fig. 4 The Pneumatic circuit diagram

1. Have large load, can be adapted to the application of high torque output.

2. quick action and quick response.

3. Good adaptability to working environment. Especially in the flammable, explosive, dusty, strong magnetic, radiation and vibration and other worse working environment, specific hydraulic, electronic, electrical control is more superior. 


\section{Improvement design}

It is the true that our device realize the basic functions of preventing the falling objects, and can reduce the falling objects' damage to people's lives. But there are still some shortcomings. For example, the quality or speed of object is too large, whether it will affect the device performance and so on

\subsection{Improvement.}

One the further research direction: to further optimize the object detection algorithm, used of HALCON to track the falling objects position and send this through the serial port.

In the detection of falling objects algorithm, we have also tried to use the HALCON software to track the location of the falling objects.

Other direction: to improve stability of control systems. It can use single chip microcomputer with better performance.

To improve the mechanical properties of the device.

\subsection{Prospect.}

1. The realization of intelligent: at present the district is mainly with the door bar, while the construction site mainly use the green protective net. All need be previously installed to play a protective role. High-altitude anti-falling automatic device only will play its role in need, in other time will be hidden, so it make the realization of the "intelligent".

2. Wide application range: with the rapid development of economy, population is more and more concentrated, buildings are more and more high, the matter about falling objects attracts more and more people's attention.

3. Significant economic benefits: anti-altitude-fall system relate to the two major electronic and machinery industries. While with this advantages of the broad application and market, it can drive the development of the two major industries.

4. Reduce the accident: at present, there is not a type of system equipment in the market, if the product develop and marketing success, the device can greatly reduce the number of accidents caused by falling objects.

5. The reasonable combination of science and technology: such as OPENCV visual processing,IPC,and the MUC this high-end products can achieve the unique features.

\section{Summary}

The project creatively put forward the idea about the high-altitude anti-falling device and have make a primary win to achieve this function through our material object. This device have synthetically used the OPENCV computer vision library and C++ language for image processing and recognition, single-chip processor is used to receive data from the computer serial port to control the solenoid valve, pneumatic structure and so on.

\section{References}

[1]. Li Chen. A study on the behavior of high altitude falling objects in urban residential environment. Urban problems. Vol. 176 (2008) No. 3, p. 028-033.

[2]. Yin Wen-sheng, Luo Yu-lin, Li Shi-qi. Camera calibration based on OpenCV. Computer engineering and design. Vol.28 NO.1, p. 197-199.

[3]. WANG Qiang. Design of target tracking system based on visual sensing network. Modern Electronic Technique. Vol. 39 NO.8, p. 88-91.

[4]. WANG Qun-feng. The Design of Micro Cylinder Based Pneumatic. Hydraulics Pneumatic\&Seals. NO4.2007, p. 47-48. 